\title{
Evaluation of the Genisys4, a Bench-Top Preclinical PET Scanner
}

\author{
Ken Herrmann ${ }^{1,2}$, Magnus Dahlbom ${ }^{1}$, David Nathanson ${ }^{1}$, Liu Wei $^{1}$, Caius Radu ${ }^{1}$, Arion Chatziioannou ${ }^{3}$, \\ and Johannes Czernin ${ }^{1}$

\begin{abstract}
${ }^{I}$ Ahmanson Translational Imaging Division, Department of Molecular and Medical Pharmacology, David Geffen School of Medicine, UCLA, Los Angeles, California; ${ }^{2}$ Department of Nuclear Medicine, Universitätsklinikum Würzburg, Würzburg, Germany; and ${ }^{3}$ Crump Institute for Molecular Imaging, Department of Molecular and Medical Pharmacology, David Geffen School of Medicine, UCLA, Los Angeles, California
\end{abstract}

The Genisys4 is a small bench-top preclinical PET scanner designed to enable imaging in biology, biochemistry, and pharmacology laboratories and imaging centers. Here, we compare its performance with that of a well-established preclinical PET scanner. Methods: Subcutaneous and lung tumor xenografts were used to compare lesion detectability and treatment responses to chemotherapy (gemcitabine) using ${ }^{18} \mathrm{~F}-\mathrm{FDG}$ PET. The size of subcutaneous xenografts (L1210 and L1210-10K leukemia cells) and lung metastases (B16 melanoma cells) was measured on small-animal CT images. Tumor ${ }^{18} \mathrm{~F}-\mathrm{FDG}$ uptake was expressed as percentage injected dose per gram. Using list-mode data, serial images of the left ventricular blood pool were used to generate time-activity curves. Results: Subcutaneous xenografts (range, 4-12 mm; mean $\pm \mathrm{SD}, 6.1 \pm 1.7 \mathrm{~mm}$ ) and lung metastases (range, $1-5 \mathrm{~mm}$; mean, $2.1 \pm 1.2 \mathrm{~mm}$ ) were detected equally well with both scanners. Tumor ${ }^{18} \mathrm{~F}-\mathrm{FDG}$ uptake measured with both scanners was highly correlated for subcutaneous xenografts $\left(r^{2}=0.93\right)$ and lung metastases $\left(r^{2}=0.83\right)$. The new Genisys 4 scanner and the established scanner provided comparable treatment response information $\left(r^{2}=0.93\right)$. Dynamic imaging sequences permitted the generation of left ventricular blood-pool time-activity curves with both scanners. Conclusion: Using subcutaneous and lung xenografts, a novel and an established preclinical PET scanner provided equivalent information with regard to lesion detection, tumor ${ }^{18} \mathrm{~F}-\mathrm{FDG}$ uptake, tumor response to treatment, and generation of time-activity curves. Thus, the Genisys 4 provides a small, efficient bench-top preclinical PET alternative for quantitatively studying murine tumor models in biology, biochemistry, and pharmacology laboratories and preclinical imaging centers.

Key Words: preclinical PET; ${ }^{18} \mathrm{~F}-\mathrm{FDG}$; tumor models; scanner performance

J Nucl Med 2013; 54:1162-1167

DOI: 10.2967/jnumed.112.114926

$\mathbf{N}$

oninvasive imaging has become a standard approach for studying mouse models of human diseases, including cancer, cardiovascular disorders, and neurologic disorders (1-3). Smallanimal PET enables repeated whole-body imaging under various

Received Oct. 1, 2012; revision accepted Dec. 27, 2012.

For correspondence or reprints contact: Johannes Czernin, UCLA, Ahmanson Translational Imaging Division, 10833 Le Conte Ave., Room AR125 CHS, Los Angeles, CA 90095-1782.

E-mail: jczernin@mednet.ucla.edu

Published online Apr. 29, 2013.

COPYRIGHT (C 2013 by the Society of Nuclear Medicine and Molecular Imaging, Inc. conditions in vivo, thus obviating the sacrifice of large numbers of animals (4). Small-animal PET has been used for metabolic phenotyping, gene expression imaging, and monitoring of therapeutic responses $(5,6)$ and is increasingly used in drug development $(7,8)$. These applications have generated considerable interest among tumor biologists (9), who have traditionally used optical imaging approaches for noninvasive preclinical imaging (10). Optical imaging has contributed significantly to preclinical in vivo sciences based on imaging reporter gene expression. As a complement to optical imaging, PET allows a wide array of biologic, biochemical, and pharmacologic assays based on the selection and availability of specific imaging probes. However, to date, preclinical PET imaging has been used primarily in imaging centers in academia and in the pharmaceutical industry, because small-animal PET scanners are expensive and their relatively large footprint precludes their use as bench-top devices in biology and biochemistry laboratories.

These limitations provided the motivation to design a low-cost, small-footprint, high-performance bench-top preclinical PET scanner that provides high counting rate sensitivity and high image quality at low injected radioactivity doses (11). With the aim of meeting these criteria, a preclinical PET scanner (Genisys4; Sofie Biosciences) comprising 4 detector plates organized in a boxlike design was developed (12). Here, we compare the performance of this novel scanner with that of the well-established Inveon (Siemens) (13) in 2 different murine tumor models. We focused on relevant subcutaneous and lung metastasis models to determine whether the 2 scanners provide equivalent information on tumor lesion detectability, tumor ${ }^{18} \mathrm{~F}-\mathrm{FDG}$ uptake, and changes in tumor ${ }^{18} \mathrm{~F}$-FDG uptake in response to treatment. In addition, we tested the feasibility of generating time-activity curves, a prerequisite for obtaining quantitative kinetic imaging data.

\section{MATERIALS AND METHODS}

\section{Scanner Characteristics}

Genisys4. The characteristics of the Genisys4 scanner have been described previously (12) and are summarized in Table 1. In brief, the Genisys 4 consists of 4 panel detectors arranged in a box geometry. Each detector has a $24 \times 50$ bismuth germanate scintillator array with crystals measuring $1.83 \times 1.83 \times 7 \mathrm{~mm}$. The crystals are coupled to 2 H8500 photomultiplier tubes (Hamamatsu) via a glass light guide. With an energy window of $150-650 \mathrm{keV}$ optimized for mouse imaging, the peak sensitivity is approximately $14 \%$ at the center of the field of view (FOV). The spatial resolution of reconstructed images was fairly uniform across the FOV. At the center of the FOV the resolution was $1.35 \mathrm{~mm}$ in full width at half maximum. At $1.5 \mathrm{~cm}$ radial off-center the resolution was $1.37 \mathrm{~mm}$, and at $3 \mathrm{~cm}$ axial off-center the resolution was $1.32 \mathrm{~mm}$. 
TABLE 1

Scanner Characteristics

\begin{tabular}{lcc}
\multicolumn{1}{c}{ Characteristic } & Inveon \\
\hline Detector material & Lutetium oxyorthosilicate \\
Crystal dimensions $\left(\mathrm{mm}^{3}\right)$ & $1.5 \times 1.5 \times 10$ \\
Transaxial FOV $(\mathrm{mm})$ & 100 & Bismuth germanate \\
Axial FOV (mm) & $1.8 \times 1.8 \times 7$ & 45 \\
Energy window (keV) & $350-650$ \\
Sensitivity $(\%)$ & 6.7 & $150-650$ \\
Reconstructed resolution $(\mathrm{mm})$ (center of FOV) & $1.8^{\star}$
\end{tabular}

*Measured on point-source images reconstructed with parameters used in routine imaging.

The scanner includes a real-time respiratory monitoring system, with monitoring and control of anesthesia and temperature for biologic stability and animal safety. Genisys 4 provides anatomic reference images via a combination of an x-ray projection, a photographic image, and a digital mouse atlas (14). The optical camera also provides visual monitoring of the mouse during the imaging procedure. The anatomic reference images are also used for attenuation correction in image reconstruction on the Genisys4 (13).

Inveon. The Inveon scanner has been described in detail previously (13), and some of the characteristics are shown in Table 1. The Inveon scanner consists of 64 lutetium oxyorthosilicate block detectors, where the size of each detector element is $1.5 \times 1.5 \times 10 \mathrm{~mm}$. It has a $16.1-\mathrm{cm}$ ring diameter, a transaxial FOV of $10 \mathrm{~cm}$, and an axial FOV of $12.7 \mathrm{~cm}$. Its sensitivity is $6.6 \%$ using an energy window of $350-650 \mathrm{keV}$. In-plane radial and tangential resolutions, reconstructed with point-spread function-based maximum-likelihood expectation maximization, was below $1.8 \mathrm{~mm}$ in full width at half maximum at the center of the FOV.

\section{Animal Experimental Studies}

All animal experiments were approved by the UCLA Animal Research Committee and were performed according to the guidelines of the Division of Laboratory Animal Medicine at UCLA.

In Vivo Models for Tumor Detection. The murine leukemic lines L1210 wild-type and L1210-10K were used to generate subcutaneous xenografts (15). L1210 cells highly express deoxycytidine kinase $(\mathrm{dCK})$, the enzyme that converts gemcitabine into its active form, whereas L1210-10K cells do not express dCK.

We used these cell lines (a gift from Charles Dumontet Université Claude Bernard Lyon I) to generate xenografts because response or nonresponse to gemcitabine was predictable $(16,17)$. Using xenografts with a wide range of $\mathrm{dCK}$ expression and activity provides wide ranges of glucose metabolic responses to gemcitabine, which in turn allowed response monitoring with both scanners across a wide range of glucose metabolic changes.

L1210 and L1210-10K were cultured in RPMI medium 1640, supplemented with $5 \%$ fetal calf serum and $2 \mathrm{mM} \mathrm{L-glutamine,} \mathrm{in}$ a $5 \% \mathrm{CO}_{2} 37^{\circ} \mathrm{C}$ incubator. $2.0 \times 10^{6} \mathrm{dCK}$-positive $\mathrm{L} 1210$ wild-type cells and $2.0 \times 10^{6} \mathrm{dCK}$-negative L1210-10K cells were implanted in the flanks of each SCID mouse. For lung metastasis studies, 250,000 B16 F/10 mouse melanoma cells were resuspended in phosphatebuffered saline and injected via tail vein in three 6- to 8-wk-old C57 BL/6 mice. Mice were repeatedly imaged with small-animal CT (Siemens Preclinical Solutions Inc.) to detect lung metastases as described below.

Tumor Detectability and Treatment Response Assessments. To create xenografts of varying sizes, baseline ${ }^{18} \mathrm{~F}$-FDG PET scans were obtained on both scanners twice (at days 2 and 4 after tumor cell implantation in 8 mice, and at days 3 and 5 after implantation in 6 mice).
Posttreatment PET scans were performed $1 \mathrm{~d}$ after gemcitabine treatment (day 5 after tumor cell implantation in 8 mice and day 6 after tumor cell implantation in another 6 mice). This protocol was selected to permit comparisons of tumor ${ }^{18} \mathrm{~F}-\mathrm{FDG}$ uptake across tumors of various sizes as well as to compare therapy-induced changes in tumor ${ }^{18}$ F-FDG uptake.

Gemcitabine was obtained from the UCLA pharmacy, and stock solutions were prepared in $0.9 \%$ saline. Gemcitabine treatment (360 $\mathrm{mg} / \mathrm{kg}$ intraperitoneally) commenced on day $4(n=8)$ or on day $5(n=6)$ after tumor cell implantation and was repeated on days $8(n=8)$ and 9 $(n=6)$, respectively.

In Vivo Lung Metastasis Model. Three mice were screened by small-animal CT for the existence of lung lesions every 3-4 d. Lung metastases became visible $17 \mathrm{~d}$ after injection of tumor cells into the tail vein. Many of these lung lesions were not measurable because they exhibited a diffuse growth pattern. Only distinct, solid, measurable lung lesions were included in the analysis. After these distinct lesions became visible, PET and small-animal CT imaging studies were performed 18 and $21 \mathrm{~d}$ after injection of tumor cells into the tail vein. This schedule was applied to ascertain a wide range of measurable lesion sizes.

Animal Preparation for Imaging. A standardized protocol was used (18). Mice were kept fasting for at least $4 \mathrm{~h}$ and were warmed on a heating pad before and after ${ }^{18} \mathrm{~F}-\mathrm{FDG}$ injection. The mean $( \pm \mathrm{SD})$ injected dose of ${ }^{18} \mathrm{~F}-\mathrm{FDG}$ was $936.1 \pm 55.5 \mathrm{kBq}(25.3 \pm 1.5 \mu \mathrm{Ci})$ for the $\mathrm{L} 1210$ mice and $936.1 \pm 18.5 \mathrm{kBq}(25.3 \pm 0.5 \mu \mathrm{Ci})$ for the B16 mice. ${ }^{18} \mathrm{~F}-\mathrm{FDG}$ was administered during isoflurane anesthesia, and the mice remained anesthetized throughout the tracer uptake period and until imaging was completed.

\section{Image Acquisition}

After an uptake period of $60 \mathrm{~min}$, the mice were placed in a dedicated imaging chamber designed for use with the CT and both PET scanners. Whole-body PET images were acquired first using the Genisys 4 scanner, and images were acquired for $10 \mathrm{~min}$. The imaging chamber was then transferred to the Inveon scanner to acquire another set of 10-min static images. Finally, the mice were transferred to the small-animal CT scanner. The CT acquisition parameters were $70 \mathrm{kVp}, 500 \mu \mathrm{A}$, and 360 views with an exposure time of $500 \mathrm{~ms}$ at each view, resulting in a soft-tissue dose of about $8 \mathrm{cGy}$ (19). A Feldkamp algorithm was used to reconstruct images.

To generate time-activity curves, dynamic images were reconstructed using the Inveon $(n=1)$ and Genisys4 $(n=1)$ scanners. Data were collected in list mode over $1 \mathrm{~h}$. At the end of the acquisition, the data were rebinned into dynamic frames $(5 \times 2 \mathrm{~s}, 10 \times 5 \mathrm{~s}$ and $1 \times 3,540 \mathrm{~s})$. The 15 initial frames were used to extract the time-activity curves from the left ventricular blood pool and the liver. The final long frame was used for region-of-interest definition. 


\section{Image Reconstruction}

For both scanners, data were corrected for random coincidences, decay, and dead time. Inveon images were reconstructed using 3dimensional ordered-subsets expectation maximization (2 iterations, 8 subsets) followed by 3 -dimensional maximum a posteriori reconstruction. For the Genisys4, maximum-likelihood expectation maximization was used to create the final image volumes, as recommended by the vendor. All images were corrected for photon attenuation; however, scatter correction was not applied. For cross-calibration between the dose calibrator and the imaging systems, a 2.5- by $6.0-\mathrm{cm}$ cylinder phantom filled with $15.5 \mathrm{kBq}(0.42 \mu \mathrm{Ci}) / \mathrm{mL}$ of ${ }^{18} \mathrm{~F}-$ FDG was imaged. From this scan, a system calibration factor was derived by dividing the known activity concentration in the phantom by the measured mean counts per voxel in the reconstructed PET images.

\section{Image Analysis}

PET, CT, and PET/CT images were analyzed using OsiriX Imaging Software (version 3.8; OsiriX). CT images were used only for size measurements and lesion localization. For the lung metastasis model, CT images were also used to determine whether lesions were distinct rather than diffuse and were therefore measurable.

The CT lung images were first reviewed with masking of the PET findings. Measurable lesions (those that could clearly be separated from normal lung parenchyma) were identified. Up to 4 measurable lesions were identified in each mouse, their size was measured with smallanimal CT, and their locations were recorded (upper, mid, or lower portion of the right or left lung). Then, all PET images acquired on the Inveon and Genisys4 were reviewed in a random sequence by an observer who did not know the scanner on which the images had been acquired or the CT findings. Lesion location and degree of uptake were recorded. Finally, lesions identified on PET were matched with measurable lesions identified on CT.

Consecutive 2-dimensional regions of interest were drawn on subcutaneous and lung lesions on coronal and axial images to detect the maximum ${ }^{18} \mathrm{~F}$-FDG uptake (percentage injected dose [\% ID]/g). These regions encompassed the entire metabolically active tumor. The same region-of-interest approach was used consistently for images derived from both scanners.

To generate the time-activity curves, a 1.5 -mm-diameter spheric region of interest was first defined at the center of the left ventricle on the last long frame on which the myocardium was well visualized. The time-activity curves were then extracted from the sequence of short image frames that were reconstructed for the first $60 \mathrm{~s}$ of the acquisition.

\section{Statistical Analysis}

Correlations between tumor ${ }^{18} \mathrm{~F}-\mathrm{FDG}$ uptake in subcutaneous and lung tumors were sought using least-squares regression analysis. Changes in tumor uptake in response to treatment were expressed as the ratio of baseline over follow-up measurements of tumor ${ }^{18} \mathrm{~F}-\mathrm{FDG}$ uptake on day 1 after treatment.

\section{RESULTS}

Subcutaneous xenografts ranged in size from 4 to $12 \mathrm{~mm}$ (mean, $6.1 \pm 1.7 \mathrm{~mm}$ ). ${ }^{18} \mathrm{~F}$-FDG uptake was depicted equally well on images from both scanners. Tumor ${ }^{18} \mathrm{~F}-\mathrm{FDG}$ uptake expressed as $\% \mathrm{ID} / \mathrm{g}$ ranged from 5.1 to 31.7 for the Inveon and from 4.6 to 42.8 for the Genisys4 ( $P=$ not statistically significant $[\mathrm{NS}]$ ). Representative images are shown in Figure 1. ${ }^{18} \mathrm{~F}$-FDG uptake measurements obtained from the 2 scanners were closely correlated $\left(R^{2}=0.93\right.$; $P<0.001 ; y=1.26 x-1.52$; Fig. 2)

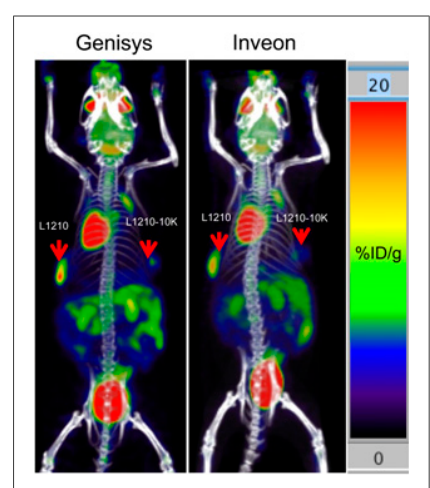

FIGURE 1. ${ }^{18} \mathrm{~F}-\mathrm{FDG} \quad \mathrm{PET} / \mathrm{CT}$ maximum-intensity projections obtained with Genisys 4 and Inveon PET scanners. L1210 and L1210-10K subcutaneous xenografts (red arrows) measured 8 and $6 \mathrm{~mm}$ in largest diameter; tumor uptake $(\% \mathrm{ID} / \mathrm{g})$ was comparable (20.4 vs. 16.0 for $L 1210$ and 7.5 vs. 6.7 for L1210-10K xenografts). were closely correlated $\left(y=1.42 x+16.3 ; R^{2}=0.93 ; P<0.001\right)$ (Fig. 4).

\section{Lung Metastases}

A total of 10 lung lesions in 4 mice (maximum of 4 lesions per mouse) were analyzed. The number of measurable lung metastases ranged from 1 to 4 per mouse. Lung lesions as small as $1 \mathrm{~mm}$ in largest diameter were identified as measurable. Measurable lesions ranged in size from 1 to $5 \mathrm{~mm}$ (mean, $2.1 \pm 1.2 \mathrm{~mm}$ ). Hypermetabolic lung lesions were detected equally well by both scanners (Fig. 5). ${ }^{18} \mathrm{~F}-\mathrm{FDG}$ uptake measurements (\% ID/g), ranging from 5.0 to 24.2 and 4.0 to $27.2(P=\mathrm{NS})$ as measured by the

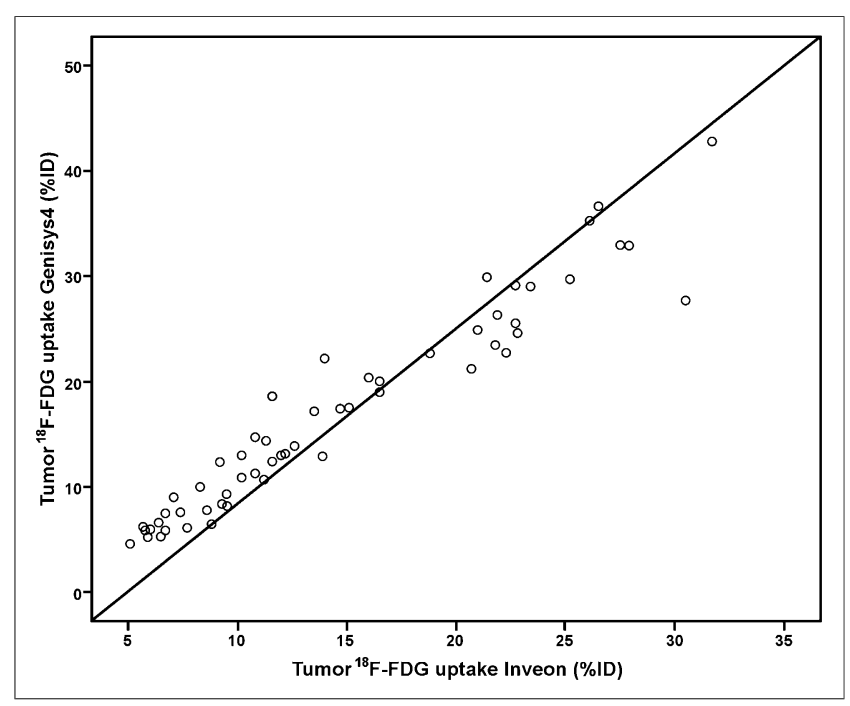

FIGURE 2. Correlation between ${ }^{18} \mathrm{~F}-\mathrm{FDG}$ tumor uptake measured with Inveon and Genisys 4 scanners was highly significant $(y=1.26 x$ - 1.52; $\left.R^{2}=0.932 ; P<0.001\right)$. 


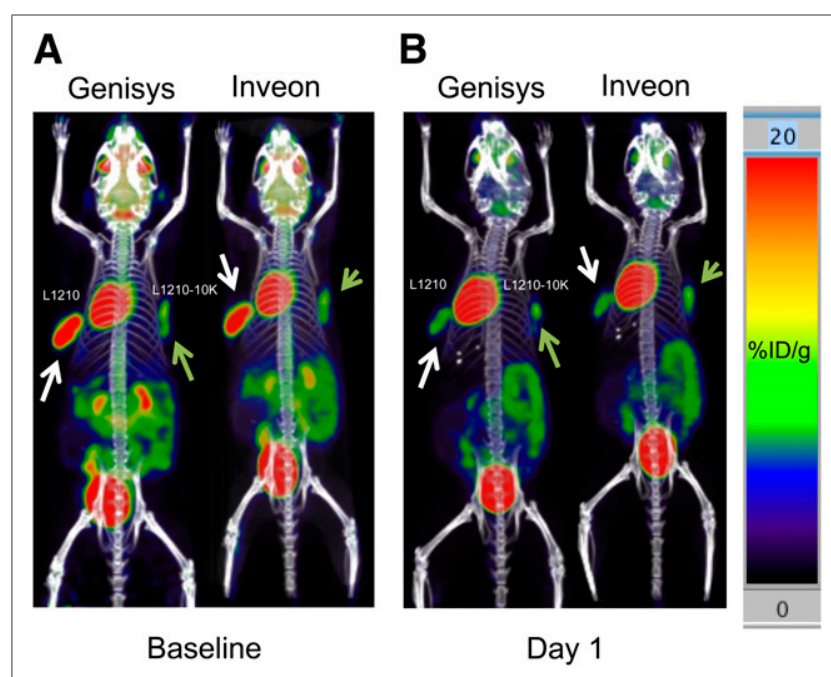

FIGURE 3. Coronal PET/CT maximum-intensity projections depict gemcitabine-sensitive (white arrows) and -resistant (green arrows) tumors. Images obtained at baseline with Genisys 4 and Inveon scanners $(A)$ and after $1 \mathrm{~d}$ of gemcitabine treatment $(B)$ are shown. Gemcitabine-sensitive xenografts responded to treatment, with significant decreases in tumor ${ }^{18} \mathrm{~F}-\mathrm{FDG}$ uptake, whereas resistant xenografts (green arrows) showed stable ${ }^{18} \mathrm{~F}-\mathrm{FDG}$ uptake.

Inveon and Genisys 4 , respectively, were closely correlated ( $y=$ $1.08 x-1.63 ; R^{2}=0.83 ; P<0.001$ ) (Fig. 6).

\section{Time-Activity Curves}

Examples of left ventricular blood-pool and liver time-activity curves generated from the 2 scanners are shown in Figure 7. Because the injected activity was approximately 3 times higher on the Inveon scanner than on the Genisys4 $(2,072$ and $629 \mathrm{kBq}$ [56 $\mu \mathrm{Ci}$ and $17 \mu \mathrm{Ci}$ ], respectively), the vertical scales are different on the 2 graphs. The curves on the 2 graphs were similar in shape. One significant difference was that the initial peak of the blood-pool curve generated from the Genisys 4 scanner was better defined, and the activity at later times decreased more pre-

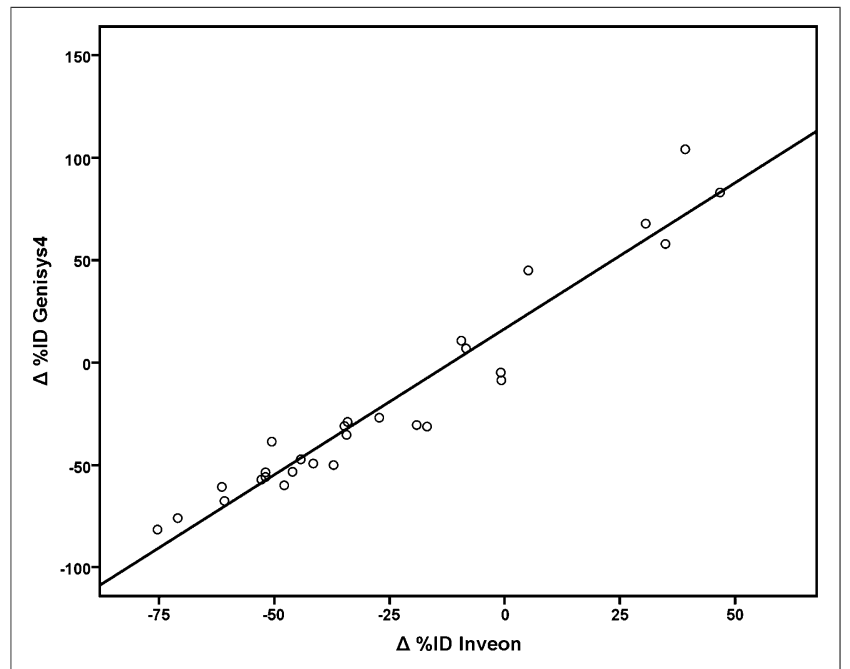

FIGURE 4. Treatment-induced changes in tumor ${ }^{18} \mathrm{~F}-\mathrm{FDG}$ uptake measured with Genisys 4 and Inveon scanners were significantly correlated $\left(y=1.42 x+16.3 ; r^{2}=0.93 ; P<0.001\right)$.

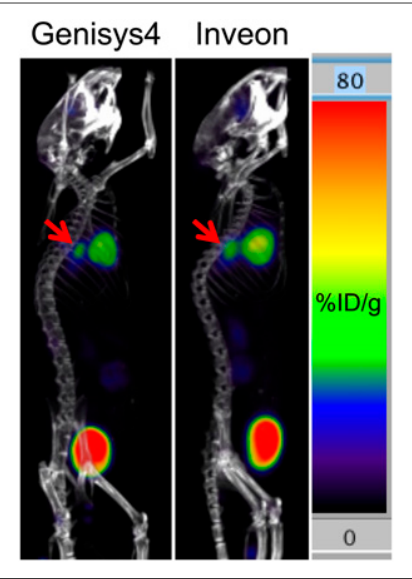

FIGURE 5. Sagittal views of 2.5 $\mathrm{mm}$ B16-melanoma lung metastasis (arrows). Tumor ${ }^{18} \mathrm{~F}-\mathrm{FDG}$ uptake measurements $(\% \mathrm{ID} / \mathrm{g})$ were nearly identical for both scanners (16.3\% and $16.4 \%$, respectively).

\section{uptake information in subcu-} taneous and lung metastasis cancer models. Furthermore, the responses of subcutaneous xenografts to gemcitabine were assessed equally well. Finally, both scanners provided measurements of the arterial input function and thus, after further refinement and validation, should allow for kinetic analysis and quantification of metabolic processes in vivo.

The interest in preclinical PET imaging using a variety of imaging probes has increased substantially over the last few years. PET imaging has been used for tumor metabolic phenotyping $(9,20)$, and ${ }^{18} \mathrm{~F}-\mathrm{FDG}$ has been used as a pharmacodynamic biomarker to assess tumor responses to drugs $(9,21,22)$. Based on the selection and availability of specific probes, protocols and findings from preclinical PET are readily and relevantly translatable into clinical trials and practice.

However, there are several impediments to the widespread adoption of preclinical PET. These include the high cost of equipment acquisition and maintenance, a large footprint requiring substantial laboratory space, and skilled personnel. There is therefore a need for introducing low-cost, high-performance preclinical PET scanners that integrate animal support functions into the imaging system and provide high-quality PET images.

Cost can be lowered by reducing the number of required detectors and the image plane diameter. This has been achieved for the Genisys 4 by using a panel detector system in a box designed for mice, since they are the most commonly used animal model today. Importantly, although the number of detectors has been decreased, the sensitivity of the system is extremely high and the box geometry allows for fully tomographic image reconstruction (12).

The large footprint of current commercial scanners precludes their placement in individual investigator's laboratories. The Genisys4 is a small, low-weight, bench-top scanner that can be placed in a biosafety-grade environment of 2 or more, thus obviating the transportation of often severely immune-compromised mice into a nonsterile environment for imaging. Even when this scanner is placed in an imaging laboratory, the small footprint should be beneficial for more efficient space use.

Because of its high sensitivity and dead-time correction limitations (23), very low doses of injected radioactivity are used for Genisys4 studies without comprising image quality and quantitative 


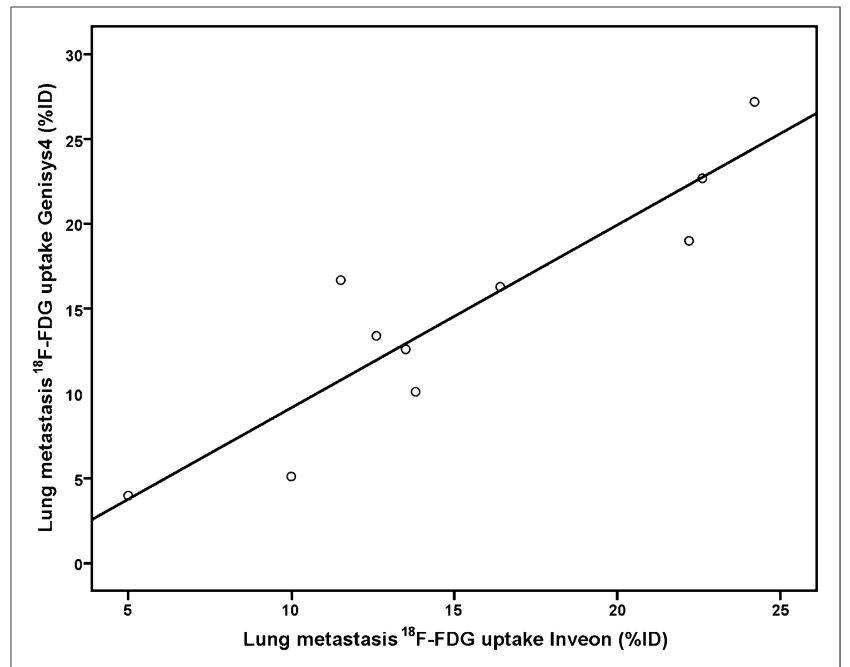

FIGURE 6. ${ }^{18} \mathrm{~F}-\mathrm{FDG}$ uptake in lung xenografts measured with Inveon and Genisys 4 scanners was closely correlated $(y=1.08 x-$ 1.63; $\left.r^{2}=0.83 ; P<0.001\right)$.

accuracy. In fact, injected doses averaged around $925 \mathrm{kBq}(25 \mu \mathrm{Ci})$ in the current study.

Obviously, the critical question is whether the performance of the new preclinical bench-top scanner matches that of an established preclinical scanner. We used several parameters to compare the performance of the 2 scanners. First, we demonstrated that the 2 scanners had identical sensitivity in detecting subcutaneous xenografts and lung metastases that ranged in size from 1 to $12 \mathrm{~mm}$ (Figs. 1 and 5).

We then tested both scanners for their ability to measure tumor responses to therapy. We used a well-established model derived from leukemia cells that either do (L1210) or do not (L1210-10K) express dCK. We used gemcitabine as the study drug because its cytotoxic effects depend on dCK activity and are thus predictable (17). Thus, only tumors with high dCK activity respond to gemcitabine treatment (Fig. 3). As expected, dCK-negative xenografts (L1210-10K) did not respond to gemcitabine, whereas significant reductions in tumor ${ }^{18} \mathrm{~F}$-FDG uptake were observed in dCK-positive xenografts derived from L1210 cell lines. The degree of changes in ${ }^{18}$ F-FDG uptake as measured with both scanners was closely correlated (Fig. 4). Tumor responses to treatment were equally well measured with both scanners.

Several limitations have to be kept in mind regarding the comparison of the 2 scanners. First, the Genisys 4 is designed to include attenuation correction without user intervention. In contrast, data acquired with the Inveon are not routinely corrected for photon attenuation. Nevertheless, the method of measuring the scanner calibration factor (with a cylinder filled with ${ }^{18} \mathrm{~F}$-FDG) essentially calibrates for this difference, since the Inveon includes the attenuation of the cylinder in the measurements. This assumes that the attenuation of the cylinder is the same as the attenuation of a mouse - which is close but would have a tendency to under-correct the activity in deeply located structures (e.g., lung lesions). Superficial structures, such as subcutaneous xenografts, would be affected to a lesser degree. Second, whereas scatter correction was not applied to the data of either scanner, scatter on the Genisys 4 is more significant because of the more compact geometry and wide energy window used $(26.1 \%$ vs. $7.1 \%(12,13))$. This lack of scatter correction together with the attenuation correction is very likely to result in overestimation of measured activity on this scanner. These 2 effects might explain the systematic difference between the slopes for lung lesions versus xenografts. The lack of scatter correction, together with the differential acceptance of scatter events due to the different energy windows, could explain the overall higher tumor uptake as measured with the Genisys4 (i.e., a slope greater than 1) than with the Inveon (Figs. 2, 4, and 6). The difference in slopes between the xenografts and the lung tumors could be attributed to the under-correction of the lung tumor uptake for attenuation as measured with the Inveon.

Third, all images were reconstructed with the manufacturers' recommended parameters, which result in a final image resolution that is slightly better on the Genisys4 than on the Inveon $(12,13)$ and will, in turn, result in a greater underestimation of activity in small tumors on the Inveon than on the Genisys4. These differences in scanner performance can account for and produce differences in the measured probe tissue concentrations between the 2 scanners.

Fourth, because of limited dead-time correction capabilities and detector counting-rate limitations, low activities need to be injected
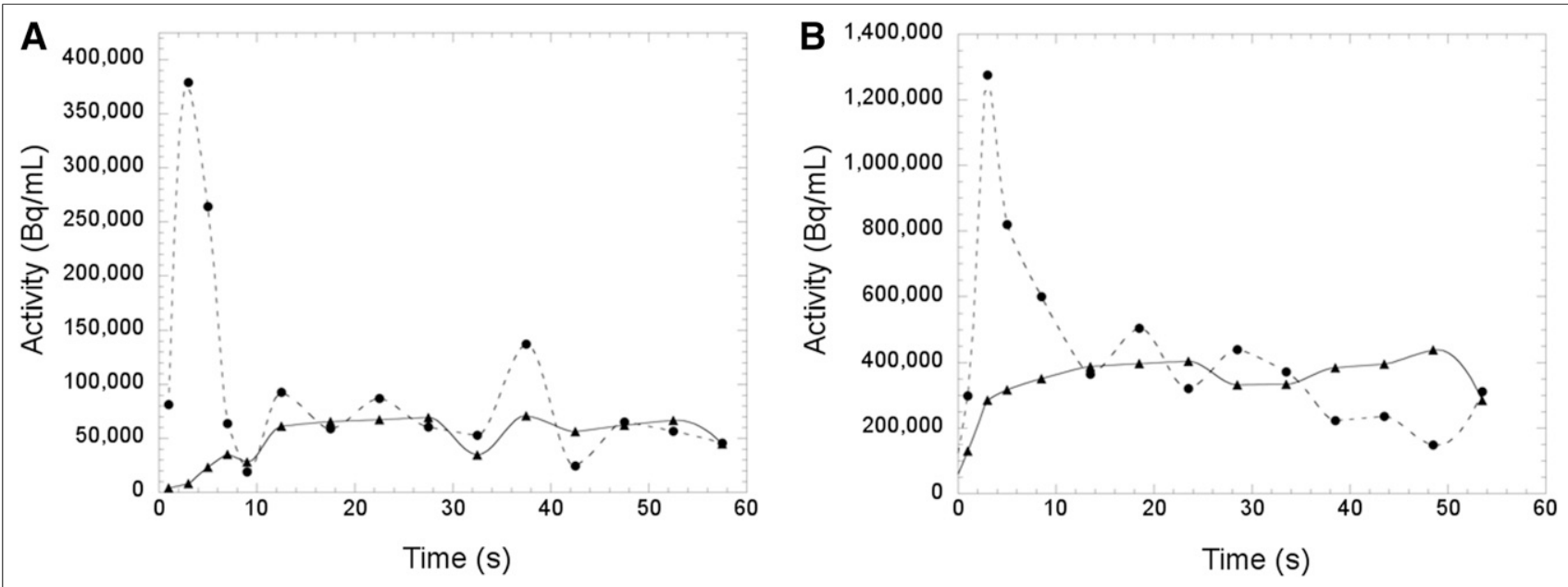

FIGURE 7. Time-activity curves were generated from both scanners by placing 1.5-mm-diameter spheric regions of interest within left ventricular blood pool and liver on serial short image frames during first $60 \mathrm{~s}$ of acquisition. Because injected activity was approximately 3 times higher on Inveon scanner than on Genisys 4 scanner (2,072 and $629 \mathrm{kBq}$ [56 and $17 \mu \mathrm{Ci}]$, respectively), vertical scales are different in A and B. 
for studies using the Genisys4 scanner. The Inveon PET studies were performed after injection of $3,700 \mathrm{kBq}(100 \mu \mathrm{Ci})$ of ${ }^{18} \mathrm{~F}-\mathrm{FDG}$. This scanner, though, is capable of working at higher counting rates, which would increase signal-to-noise ratio for short time frames. Therefore, the quality of the dynamic data acquired with the Inveon scanner would likely have been better with higher injected doses. A detailed investigation of the limits of detection for the 2 scanners is beyond the scope of this work (23).

PET imaging using appropriate tracer kinetic models can be used to quantify molecular processes in vivo (24). Such quantitative measurements require kinetic data, which in turn require scanners with a high temporal and spatial resolution. Once such dynamic data are acquired, they can be fitted to appropriate kinetic models to derive, for instance, glucose metabolic rates in $\mu \mathrm{mol} / \mathrm{g} / \mathrm{min}$ (25). Quantitative approaches have been tested and validated in humans for their ability to more accurately describe tumor metabolism and tumor responses to treatment $(26,27)$.

To determine whether kinetic data can be derived from both scanners, we acquired dynamic images with both scanners. A prior study from UCLA established the feasibility of deriving the arterial input function from serial small-animal PET ${ }^{18} \mathrm{~F}-\mathrm{FDG}$ measurements of left ventricular blood-pool activity (28). We obtained curves of similar shapes for the left ventricular blood pool and liver, suggesting that both scanners can provide such quantitative data (Fig. 7). A true validation of the data would have required arterial blood sampling to measure plasma radiotracer activity. However, such validation was beyond the scope of the current study.

\section{CONCLUSION}

We have evaluated a small-footprint, low-cost, lightweight, high-sensitivity bench-top preclinical PET scanner by comparing its performance with that of an established commercial preclinical scanner. We found a comparable performance with regard to lesion detection, tumor drug response assessments, and the ability to generate kinetic data. Because of its high sensitivity, very low injected radioactivity doses were sufficient to obtain this information. This scanner should therefore enable the use of preclinical PET imaging in biology, biochemistry, and pharmacology labs involved in drug development.

\section{DISCLOSURE}

The costs of publication of this article were defrayed in part by the payment of page charges. Therefore, and solely to indicate this fact, this article is hereby marked "advertisement" in accordance with 18 USC section 1734. Drs. Caius Radu, Arion Chatziioannou, and Johannes Czernin are founders of Sofie Biosciences, which manufactures the Genisys 4 scanner. No other potential conflict of interest relevant to this article was reported.

\section{REFERENCES}

1. Bloomfield PM, Rajeswaran S, Spinks TJ, et al. The design and physical characteristics of a small animal positron emission tomograph. Phys Med Biol. 1995;40:1105-1126.

2. Cherry SR, Shao Y, Silverman RW, et al. MicroPET: a high resolution PET scanner for imaging small animals. IEEE Trans Nucl Sci. 1997;44:1161-1166.
3. Phelps ME. Positron emission tomography provides molecular imaging of biological processes. Proc Natl Acad Sci USA. 2000;97:9226-9233.

4. Herschman HR, MacLaren DC, Iyer M, et al. Seeing is believing: non-invasive, quantitative and repetitive imaging of reporter gene expression in living animals, using positron emission tomography. J Neurosci Res. 2000;59:699-705.

5. Walter MA, Benz MR, Hildebrandt IJ, et al. Metabolic imaging allows early prediction of response to vandetanib. J Nucl Med. 2011;52:231-240.

6. Waldherr C, Mellinghoff IK, Tran C, et al. Monitoring antiproliferative responses to kinase inhibitor therapy in mice with $3^{\prime}$-deoxy- $3^{\prime}-{ }^{18} \mathrm{~F}$-fluorothymidine PET. J Nucl Med. 2005;46:114-120.

7. Toyohara J, Ishiwata K. Animal tumor models for PET in drug development. Ann Nucl Med. 2011;25:717-731.

8. Aboagye EO. Positron emission tomography imaging of small animals in anticancer drug development. Mol Imaging Biol. 2005;7:53-58.

9. Chen Z, Cheng K, Walton Z, et al. A murine lung cancer co-clinical trial identifies genetic modifiers of therapeutic response. Nature. 2012;483:613-617.

10. Pysz MA, Gambhir SS, Willmann JK. Molecular imaging: current status and emerging strategies. Clin Radiol. 2010;65:500-516.

11. Zhang H, Vu NT, Bao Q, et al. Performance characteristics of BGO detectors for a low cost preclinical PET scanner. IEEE Trans Nucl Sci. 2010;57:1038-1044.

12. Gu Z, Taschereau R, Vu NT, et al. Design and initial performance of PETbox4, a high sensitivity preclinical imaging tomograph. In: Nuclear Science Symposium and Medical Imaging Conference (NSS/MIC). Piscataway, NJ: IEEE; 2011:2328-2331.

13. Bao Q, Newport D, Chen M, Stout DB, Chatziioannou AF. Performance evaluation of the Inveon dedicated PET preclinical tomograph based on the NEMA NU-4 standards. J Nucl Med. 2009;50:401-408.

14. Wang H, Stout DB, Taschereau R, et al. MARS: a mouse atlas registration system based on a planar x-ray projector and an optical camera. Phys Med Biol. 2012;57:6063-6077.

15. Jordheim LP, Cros E, Gouy MH, et al. Characterization of a gemcitabineresistant murine leukemic cell line: reversion of in vitro resistance by a mononucleotide prodrug. Clin Cancer Res. 2004;10:5614-5621.

16. Lee JT, Campbell DO, Satyamurthy N, Czernin J, Radu CG. Stratification of nucleoside analog chemotherapy using $1-\left(2^{\prime}\right.$-deoxy-2'-18 F-fluoro-beta-D-arabinofuranosyl)cytosine and 1-(2'-deoxy- $2^{\prime}-{ }^{18} \mathrm{~F}$-fluoro-beta-L-arabinofuranosyl)-5methylcytosine PET. J Nucl Med. 2012;53:275-280.

17. Laing RE, Walter MA, Campbell DO, et al. Noninvasive prediction of tumor responses to gemcitabine using positron emission tomography. Proc Natl Acad Sci USA. 2009;106:2847-2852.

18. Fueger BJ, Czernin J, Hildebrandt I, et al. Impact of animal handling on the results of ${ }^{18}$ F-FDG PET studies in mice. J Nucl Med. 2006;47:999-1006.

19. Taschereau R, Chow PL, Chatziioannou AF. Monte Carlo simulations of dose from microCT imaging procedures in a realistic mouse phantom. Med Phys. 2006;33:216-224.

20. Lieberman BP, Ploessl K, Wang L, et al. PET imaging of glutaminolysis in tumors by ${ }^{18} \mathrm{~F}-(2 \mathrm{~S}, 4 \mathrm{R}) 4$-fluoroglutamine. J Nucl Med. 2011;52:1947-1955.

21. Fernandes E, Barbosa Z, Clemente G, Alves F, Abrunhosa AJ. Positron emitting tracers in pre-clinical drug development. Curr Radiopharm. 2012;5:90-98.

22. Hurov JB, Huang M, White LS, et al. Loss of the Par-1b/MARK2 polarity kinase leads to increased metabolic rate, decreased adiposity, and insulin hypersensitivity in vivo. Proc Natl Acad Sci USA. 2007;104:5680-5685.

23. Bao Q, Chatziioannou AF. Estimation of the minimum detectable activity of preclinical PET imaging systems with an analytical method. Med Phys. 2010; 37:6070-6083.

24. Tomasi G, Turkheimer F, Aboagye E. Importance of quantification for the analysis of PET data in oncology: review of current methods and trends for the future. Mol Imaging Biol. 2012;14:131-146.

25. Huang SC, Phelps ME, Hoffman EJ, Sideris K, Selin CJ, Kuhl DE. Noninvasive determination of local cerebral metabolic rate of glucose in man. Am J Physiol. 1980;238:E69-E82.

26. Dimitrakopoulou-Strauss A, Strauss LG, Egerer G, et al. Impact of dynamic ${ }^{18} \mathrm{~F}-$ FDG PET on the early prediction of therapy outcome in patients with high-risk soft-tissue sarcomas after neoadjuvant chemotherapy: a feasibility study. $\mathrm{J} \mathrm{Nucl}$ Med. 2010;51:551-558.

27. Muzi M, Spence AM, O'Sullivan F, et al. Kinetic analysis of $3^{\prime}$-deoxy- $3^{\prime}-{ }^{18} \mathrm{~F}-$ fluorothymidine in patients with gliomas. J Nucl Med. 2006;47:1612-1621.

28. Ferl GZ, Zhang X, Wu HM, Kreissl MC, Huang SC. Estimation of the ${ }^{18}$ F-FDG input function in mice by use of dynamic small-animal PET and minimal blood sample data. J Nucl Med. 2007;48:2037-2045. 